\title{
BMJ Open Efficacy of cognitive behavioural therapy-based smartphone app for smoking cessation in China: a study protocol of a randomised controlled trial
}

Yanhui Liao (D) , ${ }^{1,2}$ Jinsong Tang ${ }^{1,2}$

To cite: Liao Y, Tang J. Efficacy of cognitive behavioural therapy-based smartphone app for smoking cessation in China: a study protocol of a randomised controlled trial. BMJ Open 2021;11:e041985. doi:10.1136/ bmjopen-2020-041985

- Prepublication history and additional material for this paper is available online. To view these files, please visit the journal online (http://dx.doi.org/10. 1136/bmjopen-2020-041985).

Received 22 June 2020 Revised 07 December 2020 Accepted 29 December 2020

Check for updates

(C) Author(s) (or their employer(s)) 2021. Re-use permitted under CC BY-NC. No commercial re-use. See rights and permissions. Published by BMJ.

${ }^{1}$ Department of Psychiatry, Sir Run Run Shaw Hospital, School of Medicine, Zhejiang University, Hangzhou, China

${ }^{2}$ Key Laboratory of Medical Neurobiology of Zhejiang

Province, Hangzhou, China

Correspondence to

Dr Yanhui Liao;

liaoyanhui@zju.edu.cn

\section{ABSTRACT}

Introduction A number of smartphone apps have been proved to be effective in assisting smoking cessation in many countries, but little is known about the effectiveness of smartphone apps that assist in smoking cessation in China. This study aims to evaluate the efficacy of cognitive behavioural therapy (CBT)-based cigarette smoking cessation smartphone app for treatment-seeking smokers in China.

Methods and analysis In this remote control, randomised controlled trial, the participants will be randomised and allocated in 1:1 ratio to smoking cessation app intervention group or to control group. Participants will receive a 12week intervention (treatment group will receive CBT-based cigarette smoking cessation smartphone app intervention and control group will receive text messaging-based control intervention) with follow-up at week 26 . The primary outcome includes biologically verified continuous smoking abstinence at week 26 after the quit date. The main secondary outcomes include 7-hour point prevalence smoking abstinence at weeks 1, 2, 3, 4, 8, 12, 16, 20 and 26 , self-reported continuous smoking abstinence at weeks 4, 8, 12, 16, 20 and 26 after the quit date. About 1200 smokers with willingness to make a quit attempt within a month from January 2021 to August 2021 or until the recruitment process is complete will be recruited. The final 26-week follow-up will be completed in August 2022. The trial results will be available by the end of 2022. All analyses will be done on an intention-to-treat basis. Based on the previous studies, we hypothesised that this CBTbased app will be an effective, feasible and accessible smoking cessation app in China.

Ethics and dissemination Ethical approval was obtained from the ethics committee of Sir Run Run Shaw Hospital, an affiliate of Zhejiang University, Medical College (number: 20200129-33). Study results will be disseminated via research conference presentations and peer-reviewed publications.

Trial registration number NCT04421170.

\section{INTRODUCTION}

Smoking is the leading cause of preventable morbidity and mortality throughout the world, especially in China with approximately 316 million smokers, and this accounts for nearly one-third of the world's total smokers. ${ }^{1-3}$
Strengths and limitations of this study

- The smoking cessation app is the first to be developed based on the theoretical frameworks guided (cognitive behaviour theory), evidence-based randomised controlled trial by adhering to the guideline recommendations in mainland China.

- If this app programme is proved to be effective, then it will have a huge potential use in China, wherein there are more than 300 million smokers, at population-level, and the public health impact could be enormous.

- Abstinence will be mainly measured via self-report through app questionnaires, which is less rigorous than a biochemically verified abstinence. Thus, this study will provide biological verification for those individuals who have self-reported continuous smoking abstinence at 6 months and chose this as the primary outcome in this study.

- Participants in this study will be more likely motivated than other smokers, and they can search for other smoking cessation apps or any other available smoking cessation services during the trial.

- Participants can drop out from the trial at any time point, as this smoking cessation app programme provides only online services.

In October 2016, China has announced a new national strategy, that is, Healthy China 2030, which involved decrease in cigarette smoking rate to $20 \%{ }^{4}$ The WHO Framework Convention on Tobacco Control has taken effective measures to control tobacco use and provide adequate treatment for tobacco dependence. However, smoking cessation rates remain low in China. ${ }^{5}$ Insufficient smoking cessation services are one of the most important contributing factors for the low cessation rates that have been reported in China. ${ }^{6}$ Our previous randomised controlled trial (RCT) of text-messaging-based smoking cessation intervention ('Happy Quit') has shown an increased biochemically verified continuous smoking abstinence at week 24 from around 
$2 \%$ to more than $6 \% .{ }^{7}$ The provision of more comprehensive interventions, such as widely accessible online treatment strategy for smoking cessation, might further increase the quitting rates.

So, finding more effective strategies for treatmentseeking cigarette smokers to quit smoking has become a priority due to high smoking rates in China. With increasing popularity of smartphone usage in Chinese general population, mobile devices have become the most common ones in healthcare settings for promoting health, and this led to a rapid growth in the development of medical software applications (hereafter referred to as 'apps') for these platforms. These smartphone apps provide support at a very low unit cost for those individuals who wish to quit smoking. It has become a promising way for smokers, especially the new generation smokers who might be more open to receive cessation support via digital technologies.

Currently, there are numerous apps available that assist in smoking cessation, but most of these did not adhere to the clinical practice guidelines for treating tobacco use and dependence ${ }^{8}$ and did not demonstrate clinical efficacy. ${ }^{9}$ These might be because of the 'low-certainty evidence of the benefit of smartphone apps interventions' according to a Cochrane Systematic Review. ${ }^{10}$ During the past decade, a variety of smartphone apps (approximately 70 apps) have been developed for smoking cessation in China. However, few of these have adhered to the China Clinical Smoking Cessation Guidelines (issued in 2007 and updated in 2015), and these are outlined directly from the US Clinical Practice Guideline for Treating Tobacco Use and Dependence. ${ }^{11}$ These available Chinese apps neither referred the user to a recommended treatment nor connected the users to anything outside the app, like quitline counselling or approved medications. However, the content of these Chinese apps is primarily limited and more than $80 \%$ of apps were downloaded for less than 10000 times. $^{12}$

By integrating with the guidelines and other evidencebased practices in a better manner, several studies have indicated that smoking cessation apps are effective ways for reducing smoking prevalence, especially among young adults. ${ }^{13-16}$ As China has huge cigarette smoking population with very limited evidence-based smoking cessation interventions, development of an evidence-based app that adheres to the guidelines acts as a powerful tool for smoking cessation. Furthermore, according to a survey conducted, a majority of healthcare providers have agreed that mHealth apps hold promising features for individuals to quit smoking and would be recommended to their clients, if the programme was empirically validated. ${ }^{17}$

Smoking behaviour is an unhealthy behaviour that can be changed, but changing the behaviour of people to reduce smoking is not an easy thing. Performance, stress and body weight are three main factors that influence the smoking behaviour of the people. ${ }^{18}$ Cognitive behavioural therapy (CBT) is one of the most common and the best-studied forms of psychotherapy that involves changing maladaptive thinking patterns and the negative behaviours associated with them. ${ }^{19}{ }^{20} \mathrm{CBT}$ is an effective way to treat cannabis and nicotine addiction but is less effective in treating opioid and alcohol addiction. ${ }^{20} \mathrm{CBT}$ is used as a psychological intervention for people who want to quit smoking by changing and restructuring their thought processes and its associated behaviours, which in turn assists in effective smoking cessation and prevents relapse. The training of cognitive behavioural skills assists cigarette smokers to change their smoking behaviour into no-smoking behaviours and restructuring their thought processes. It involves new learning behaviours to effectively quit smoking, maintain abstinence, ${ }^{21}{ }^{22}$ reduce weight concerns ${ }^{23}$ and cope with depression. ${ }^{24}$

This RCT evaluates the efficacy of smoking cessation smartphone app, which is a Mandarin smoking cessation app that integrates smoking cessation support and social skills training into cognitive behavioural principles and behaviour change techniques, in China. Based on the efficacy of previous smoking cessation app studies in other countries ${ }^{13-15}$ and mobile phone-based text message interventions (such as 'Happy Quit', based on the CBT) for smoking cessation in China, ${ }^{7}$ this CBT-based app is hypothesised to be an effective, feasible and accessible smoking cessation app in China. Also compared with the control app group, the rate of biologically verified continuous smoking abstinence at week 26 after the quitting date will be substantially higher in the CBT-based cigarette smoking cessation app group.

\section{METHODS AND DATA ANALYSIS}

\section{Participants and public involvement}

Neither the participants nor the public will be involved in the design, recruitment or conduction of this study.

\section{Study design and participants}

This internet-based RCT will be conducted in China. About 1200 smokers (a smoker is defined as a person who smoked more than 100 cigarettes in their lifetime, and currently smoke 5 or more cigarettes a day) who are willing to make a quit attempt within a month will be randomly allocated using a randomisation method of Interactive Web Response System (IWRS). The smokers based on balance for age (25-34 vs $>34$ years) and cigarettes per day (5-20 vs $>20$ cigarettes per day) will be randomly allocated to smoking cessation app that comprises of motivational messages and cognitive and/ or behavioural change support or to the control group that receives only text messages of thanking them for being part in this trial and reminding them till their free month at the end of follow-up. Only the participants in the intervention group will receive 12-week intervention programme. These participants will be recruited from January 2021 to August 2021 or until the recruitment process is completed. The final 26-week follow-up period will be completed by August 2022. All trial results will be 


\section{Box 1 Study inclusion and exclusion criteria}

\section{Inclusion criteria:}

1. Cigarette smokers (smoked more than 100 cigarettes in their lifetime, and currently smoke 5 or more cigarettes a day).

2. 25 years of age or older.

3. Being able to read and write in Chinese.

4. Owning a smartphone*.

5. Have experience of using apps.

6. Expressing an interest in quitting smoking within the next month.

7. Willingness to use only the study app during the course of the study.

8. Willing to provide informed consent to participate in the study.

\section{Exclusion criteria:}

1. Non-smokers.

2. Only use electronic cigarettes.

3. Smokers without attempts to quit smoking.

4. Participants with severe mental illnesst.

5. Participants who had already started their quit attempt or using any smoking cessation treatment at the time of registration.

6. Unable to use smartphone and apps.

7. Unable to read and write in Chinese.

*Smartphone operating system iOS V.9.0 or above; Android V.5.0 or above. Only subjects with iOS smartphone will be recruited during pilot phase.

†Any individuals with psychiatric symptoms that are debilitating that their ability to participate in this trial is severely impaired. These illnesses include schizophrenia, schizoaffective disorder, bipolar disorder and major depression.

available by the end of the year 2022. The overview of the eligibility criteria of the participants is described in box 1 .

\section{Sample size and power calculation}

Sample size calculations will be done based on the differences in the objective measures of the primary outcome event, that is, biologically verified continuous smoking abstinence at week 26 between intervention and control groups. Based on our previous study of 'Happy Quit', a ratio of 1:1 will be assumed for intervention to control participants, with an alpha of 0.05 , power of $80 \%$ and an effect size equal to $7 \%$ in the intervention versus $2 \%$ in the control condition, the required sample size is 486 per group with a total of 972 participants by using a two-tailed test on proportions. Considering a $20 \%$ lost to follow-up in this study, ${ }^{7}$ the final required sample size is 600 per group with a total of 1200 participants in both the groups. We planned to assess a preliminary result on 200 participants in a pilot study with 1:1 ratio (100 participants in the intervention group and 100 participants in the control group).

\section{Recruitment}

Online advertising will be used to recruit potential participants. This service will be advertised online (such as Weibo or other websites, WeChat, QQ et al) and offline (such as universities, companies, communities, hospitals). Potential participants will register by sending text messages or making a call. The research assistants will then contact the respondents to assess their eligibility and explain the study to each participant and inform them that they will be allocated to either a control group or an app programme. Baseline data and outcome data will be collected by Electronic Patient Reported Outcomes (ePRO) software (http://www.c-path.org/programs/ pro). Informed consent will be electronically obtained from each participant using an e-consent system before collecting the baseline data. Participants enrolled in this study can be withdrawn at any point of time. The participants will be asked to provide a mobile phone number for baseline assessment and follow-up as well as for receiving mobile phone-based payment as reward.

\section{Randomisation and group allocation}

The eligible participants who provide their consent to participate in the trial and have completed their baseline assessment will be randomly assigned to either intervention or control group. Randomisation (by IWRS) will be based on 1:1 ratio at individual level by balancing sex, age, level of educational, previous quit attempts and smoked cigarettes per day. The study investigators will be blinded to the participants' treatment allocation until all data have been collected. The investigators assessing the outcomes will be blinded to the participants' allocated groups until all data are analysed.

\section{Development of CBT-based cigarette smoking cessation smartphone app}

This CBT-based cigarette smoking cessation smartphone app will be developed by Johnson \& Johnson pharmaceutical company, supported by Neusoft Corporation and supervised by the Department of Psychiatry, Sir Run Run Shaw Hospital, School of Medicine, Zhejiang University. The content of the intervention will be developed based on the principles generated by the CBT and the clinical experience, scientific or empirical evidence of smoking cessation.

Eligible participants will download the app through their smartphones and activate the app by entering the code. In order to provide tailored information, such as motivational message-sending recommender, to each participant based on their personal information, their basic and smoking-related information including age, gender, years of smoking, number of cigarettes per day and motivation and self-confidence regarding smoking cessation will be entered into the app.

\section{Theory}

This CBT-based app programme integrates cognitive behavioural principles and behaviour change techniques into individualised smoking cessation support and social skill training. The aim of this app programme involves reconversion of smoking-related thoughts, knowledge and behaviour related to smoking, and it correspondingly changes smoking behaviour into stop smoking behaviour of cigarette smokers. For example, CBT technical will be used to address the smokers' concerns with regards to smoking/nicotine enhancement in the aspect 
Table 1 App features that could enhance ease-of-use

\begin{tabular}{|c|c|}
\hline Feature & Brief description \\
\hline Navigation & $\begin{array}{l}\text { Ease of navigation; users will directly } \\
\text { navigate the menu choices }\end{array}$ \\
\hline Displays & $\begin{array}{l}\text { Simplified pleasing displays with light } \\
\text { green colour }\end{array}$ \\
\hline Aesthetics & $\begin{array}{l}\text { Keep main pages simple and visually } \\
\text { appealing and encourage and make it } \\
\text { easy to use }\end{array}$ \\
\hline Minimum text & Keep text as brief as possible \\
\hline Text formatting & $\begin{array}{l}\text { Use simple and easy to understand } \\
\text { sentence, use plenty of headings, keep } \\
\text { paragraphs short, use bulleted lists and } \\
\text { highlight key terms }\end{array}$ \\
\hline Easy-to-read & $\begin{array}{l}\text { Reading level to age } 14 \text { (education of } \\
\text { middle school level) }\end{array}$ \\
\hline Language & Clear and consistent \\
\hline Font size & No small text \\
\hline Ul button setting & $\begin{array}{l}\text { Ul button setting design conforms to } \\
\text { user habits }\end{array}$ \\
\hline
\end{tabular}

UI: user interface

of performance and reduced stress or concerns of weightgain, rather than focusing on preventing the weight gain itself.

\section{App features}

The design of the app will primarily focus on the ease of use of features to promote engagement and evidencebased smoking cessation practice to improve the abstinence rates of cigarette smokers. A cigarette smoking end-user team will be invited to verify the ease of use and engagement. The overview of the app features that could enhance the ease of use is shown in table 1 and the app features that could promote engagement are shown in table 2.

\section{Summary of main features}

This CBT-based app involves a scientific-based approach with evidence-based techniques to help the participants quit smoking for good. The summary of the main features of the app are as follows:

1. CBT-based techniques for smoking cessation.

2. Create a personalised plan to help participants quit smoking for good.

3. Choose an approach that works the best for participants: either going cold turkey or slowly decreasing their nicotine intake.

4. Track the participants' cigarette consumption and their cravings.

5. Track money saving.

6. The app is built-in family and friends' support.

7. Relaxation techniques to reduce stress.

8. Multiple ways to deal with craving to smoke.

9. Motivational tips.

10. Health achievements (from their blood circulation and oxygen levels to their senses of taste and smell).

11. Emergency SOS to keep participants smoke-free for good. Multiple options will be provided to deal with them.

\section{Table 2 App features that could promote engagement}

\begin{tabular}{|c|c|}
\hline Feature & Brief description \\
\hline Gamification & Attractive gamified quitting journey with continuity story line and variable plot \\
\hline Personalisation & $\begin{array}{l}\text { Make the app as interactive as possible (eg, tailored feedback); promote engagement } \\
\text { by customised message push notification based on users' own smoking behaviour }\end{array}$ \\
\hline Professionalisation & Make the app looks professional with scientific endorsed content \\
\hline Personification & Establish a 'rapport' between the participants and healthcare providers \\
\hline Transparency and realistic expectations & Set up clear expectations concerning how the app will be used \\
\hline Shaping & Keep demands of the participants to a minimum \\
\hline Design for curiosity & $\begin{array}{l}\text { Unluck some information each day during preparation; present new information each } \\
\text { time the app is accessed }\end{array}$ \\
\hline Visual cues and dashboards & Use as many as possible images to convey information \\
\hline Structure sections & Break complex tasks into small steps \\
\hline Session time & Keep login sessions brief (each session takes less than 1 min of the users' time) \\
\hline
\end{tabular}




\section{Evidence-based smoking cessation context in app}

The content in app involves CBT targets of both smokingrelated cognitive and behavioural problems by fully integrating cognitive and behavioural strategies. The content will be guided by the cognitive behavioural theoretical frameworks and is adapted from the text messaging-based smoking cessation intervention ${ }^{7}$ and other evidence-based smoking cessation interventions ${ }^{125}$ as well as information from a survey conducted on design and development of smoking cessation apps. ${ }^{17}$ Based on this survey, the following information will be obtained from the smokers: sharing the process of smoking cessation with family members and friends (67.7\%), help them track their progress $(66.8 \%)$ and help them with their withdrawal symptoms or side effects of medications $(63.0 \%){ }^{17}$

The content contains two major parts:

1. A questionnaire including the data about demographics (gender, age, height and weight for calculating body mass index (BMI), levels of education), smoking behaviour scale of Fagerström Test for Cigarette Dependence (FTCD), previous use of smoking cessation apps and general use of health-related apps.

2. Theoretical framework guide, wherein it includes the evidence-based information with regards to smoking cessation. The app programme content will be based on cognitive behavioural intervention techniques and smoking cessation guidelines that focus on education, cognitive restructuring, self-monitoring and practical coping strategies to increase the participants' self-efficacy for successful smoking and eventually increasing the abstinence rates. CBT will be performed based on the thoughts/thinking guide of the cigarette smoker's behaviour. For example, illogical beliefs like enhanced smoking performance (cognitive process) leads to dysfunctional behaviour of continued cigarette smoking (behavioural action). To acquire the capability of quitting smoking, individuals need to recognise, understand and change their smoking behaviour patterns of monitoring their smoking patterns, identify high-risk situations, adapt the coping responses to life stressors and risky situations. The examples of types of information included in different phases of cognitive behavioural smoking cessation app programme are shown in online supplemental table 1. The information in smoking cessation app will adhere to the recommended smoking cessation practices that are presented in table 3 .

\section{Intervention}

First, the research investigators will assess each of the eligibility of each participant. At baseline assessment, all eligible participants will be invited to complete a questionnaire that contains items on demographic and smoking-related characteristics such as previous quit attempts, current smoking behaviour, motivation and determination to quit, smoking dependence by ePRO software. After completing the baseline questionnaire,
Table 3 App context adherence to smoking cessation clinical guidelines

\begin{tabular}{ll}
\hline Guideline recommended context/5 As & Yes $(\sqrt{ })$ \\
Ask $(\mathbf{X})^{\star}$
\end{tabular}

Assess

Assess the user's motivation, confidence and readiness to quit.

Assess the user's nicotine dependence and cravings.

Assist

Set and pick a quit date

Users will make a quit smoking ceremony and sign stop smoking contracts

Users will receive support for/feedback on their quit attempt

Support for increasing self-awareness of smoking and quitting by monitoring daily smoking behaviours

Information about health risks of smoking

Check progress and provide reward options Reminders about money saved since quitting

Reminders about number of cigarettes not smoked $\checkmark$ since quitting

Distraction from smoking urges, such as relaxation $\checkmark$ practice

Reminders about social benefits and health benefits accrued

\begin{tabular}{l} 
Users could interact with healthcare providers for \\
support \\
$\begin{array}{l}\text { Referral to family and friends support } \\
\text { Reminders of their own motivations during difficult } \\
\text { times }\end{array}$ \\
$\begin{array}{l}\text { Enhance motivation: risks, roadblocks, rewards } \\
\text { Informational material will be displayed }\end{array}$ \\
Links to resources were given \\
\hline
\end{tabular}

Continued 


\begin{tabular}{|c|c|}
\hline Guideline recommended context/5 As & $\begin{array}{l}\text { Yes }(\sqrt{ }) \\
\text { no }(X)^{\star}\end{array}$ \\
\hline Discussion about pharmaceutical products & $\mathrm{X}$ \\
\hline Referral to quitline or other professional support & $\mathrm{X}$ \\
\hline Support for craving and withdrawal symptoms & $\checkmark$ \\
\hline \multicolumn{2}{|l|}{ Arrange follow-ups } \\
\hline Checked-in prior to and after quit attempt & $\checkmark$ \\
\hline Support for high-risk situations & $\checkmark$ \\
\hline Support for craving and weight concern & $\checkmark$ \\
\hline Support for lapse and relapse prevention & $\checkmark$ \\
\hline If relapsed, encourage user to set a new quit date & $\checkmark$ \\
\hline $\begin{array}{l}\text { If relapsed, offer encouragement that quitting takes } \\
\text { practice }\end{array}$ & $\sqrt{ }$ \\
\hline Support identity change & $\sqrt{ }$ \\
\hline Reward and support long-term abstinence & $\sqrt{ }$ \\
\hline
\end{tabular}

5 A's: Ask, Advise, Assess, Assist, and Arrange

${ }^{*}(X)$ This information will not be provided until the end of clinical trial.

eligible participants will be automatically randomised to either intervention app or control app. The questionnaire will take about 5-10 min to complete. Randomisation will be done by Electronic Data Capture (EDC) software. The participants from both groups will be motivated to set a quit date at 1 week. The participants will receive a programme (prequit: 1 week and postquit: 12 weeks) of either personalised information (intervention group) by smartphone app or control information (control group) by mobile text messaging (short message service (SMS)) from the day after completing the questionnaire, and then will be followed up until week 26 postquit date. Both groups will have no restriction to seek other cessation methods.

\section{Control group}

For participants in the control group, SMS will be sent as are minder to fill out the self-report questionnaire via ePro system until week 26 after the quit date. After this period, the SMS will acknowledge and recommend participants to receive intervention by app for free if they want. The participants who are allocated to the control group will be encouraged to make a quit attempt and select a quit date at 1 week after completing the baseline questionnaire. They will not receive any other smoking cessation information and will receive information of thanking them for being in the study and reminding them of the time until their free month at the end of follow-up. To measure the outcomes between the two groups, continuous smoking abstinence, point prevalence of abstinence, how many cigarettes per day during the last week if they are still smoking will be checked at the end of weeks $1,2,3,4$, $8,12,16,20$ and 26 points after the quit date by ePRO software. Biochemically verified continuous abstinence will also be checked if they have reported continuous smoking abstinence at week 26 after the quit date.

\section{Intervention group}

The participants in the intervention group will receive CBT-based smoking cessation and details about the intervention app as shown in '2.5 Development of CBT-based cigarette smoking cessation smartphone app'. This will provide both mandatory information of evidence-based and guideline-based smoking cessation interventions, and optional information on quitting benefits, tips for quitting and so on. The app will provide the participants (1) with several tasks/information each day, (2) free to view any information, (3) automatic notifications at different stages of quitting process, such as daily motivational messages, tips for craving, (4) participants can also seek for help, (5) encouragement to monitor and record their smoking behaviour or write quitting diaries (eg, craving triggers), (6) to include a quitting benefits tracker that will notify the user about the health benefits and financial benefits gained after quitting and (7) to monitor their daily usage data. The app will be available for participants in the intervention group until week 26 postquit date follow-up. After this period, the app will automatically stop collecting the data but can be used if they wish to. The smoking cessation information will be tailored according to the responses provided by the participants in the smoking questionnaire and include review of their personal smoking behaviour, remind their reasons for quitting, tips to support their quit attempt, encourage to increase their motivation and boost their self-confidence, support them on how to cope with high-risk situations when they are tempted to smoke and remind them to get social support. More information will be provided during the preparation phase and early postquit phase (the first 4 weeks, especially the first week). As the participant's progress throughout the study, smoking cessation-related information will be gradually reduced until week 12 after quit date, and follow-up messages will be sent at weeks 16 , 20 and 26 after the quit date. If the participants have any questions on using the smoking cessation app, they can also seek for help (from Dr Liao, Dr Tang or research assistants, will be documented) at any time by text or WeChat or make a phone call.

\section{Outcomes}

1. Primary outcome:

a. Biologically verified continuous smoking abstinence at week 26.

2. Secondary outcomes:

a. Seven-day point prevalence of smoking abstinence at weeks 1, 2, 3, 4, 8, 12, 16, 20 and 26 follow-up after the quit date.

b. Self-reported continuous smoking abstinence at weeks 4, 8, 12, 16, 20 and 26.

c. Reduction in the number of cigarettes smoked per day.

d. Rates of participation in and completion of smoking cessation programmes. 
e. Programme acceptability will be assessed at week 4 postquit (pilot phase).

\section{Measures}

1. Biologically verified continuous smoking abstinence: Participants in this study will be considered to be biologically verified continuous smoking abstinence if they report smoking of no more than five cigarettes since the quit date and have a urine cotinine cut-off point of $200 \mathrm{ng} / \mathrm{mL}$ (cotinine urine dipsticks will be mailed to each participant's address, and test will be confirmed by video call and by family members) at week 26 (6 months) after the quit date. This abstinence has been applied by the Society for Research on Nicotine and Tobacco (SRNT) ${ }^{26}$ and the 'Russell Standard'. ${ }^{27}$

2. Seven-day point prevalence of smoking abstinence: participants who did not even have a puff of smoke for the last 7 consecutive days, and at weeks 1, 2, 3, 4, 8, 12, 16, 20 and 26.

3. Self-reported continuous smoking abstinence: a selfreport of smoking $\leq 5$ cigarettes from the past till weeks 1, 2, 3, 4, 8, 12, 16, 20 and 26.

4. Cigarettes smoked per day: number of cigarettes smoked per day within 7 days or the approximate number of cigarettes smoked within 7 days if not smoked daily.

5. Reduction in the number of cigarettes smoked per day: will be assessed by comparing the number of cigarettes smoked per day at baseline and at week 26 .

6. Nicotine dependence: nicotine dependence will be measured by the Chinese version of the FTCD. Nicotine dependence is classified using the FTCD score with the following categories: very low/none dependence (FTCD score: $<4$ ), low to medium dependence (FTCD score: 4-6) and high dependence (FTCD score: $>6) .^{728-30}$

7. Smoke craving: smoke craving will be measured by visual analogue scale (VAS, $0-10$ integers), which includes measurement of craving for a cigarette.

8. Self-efficacy: self-efficacy will be measured by VAS measurements of participants' readiness and confidence to quit smoking.

9. Motivation to quit: motivation to quit will be assessed by VAS measurements of participants' motivation to quit smoking.

10. Programme acceptability: programme acceptability will be evaluated by questions for assessing programme acceptability in table 4 . A small sample (approximately 100 participants) will be assessed at week 4 after the quit date by ePRO software.

Feasibility will also be measured by the following:

- Recruitment rate: whether we will able to recruit the targeted sample size of 1200 participants in the time allotted in this project, which will be within 6 months.

- Dropout rates: whether we will be able to achieve the follow-up rates that are high enough to conduct a larger RCT (with approximately 20\% loss to
Table 4 Questions for assessing 'CBT-based smoking cessation app' programme acceptability

\begin{tabular}{|c|c|}
\hline Question & Rating \\
\hline 1. Overall rating of the programme & $\begin{array}{l}\text { Like very much } \\
\text { Like somewhat } \\
\text { Neutral } \\
\text { Dislike somewhat } \\
\text { Very dislike }\end{array}$ \\
\hline $\begin{array}{l}\text { 2. Appraisal of 'CBT-based smoking } \\
\text { cessation app' programme: } \\
\text { likelihood of recommending programme } \\
\text { to others }\end{array}$ & $\begin{array}{l}\text { Very likely } \\
\text { Somewhat likely } \\
\text { Neutral } \\
\text { Unlikely } \\
\text { Not at all likely }\end{array}$ \\
\hline $\begin{array}{l}\text { 3. The experience of using app: ease- } \\
\text { of-use } \\
\text { 4. The experience of using app: } \\
\text { promoting engagement } \\
\text { 5. The programme made it easier to } \\
\text { quit smoking } \\
6 \text {. The programme is not the one I } \\
\text { was expected } \\
7 . \text { I would not have been able to quit } \\
\text { without the programme } \\
\text { 8. I stopped using it by the end of the } \\
\text { programme } \\
9 \text {. information was easy to understand } \\
\text { 10. information was useful for me } \\
\text { 11. The information mentioned about } \\
\text { what I was experiencing and feeling } \\
\text { 12. I received too much information }\end{array}$ & $\begin{array}{l}\text { Strongly agree } \\
\text { Agree } \\
\text { Neutral } \\
\text { Disagree } \\
\text { Strongly disagree }\end{array}$ \\
\hline 13.Frequency of using app & $\begin{array}{l}\text { Almost never } \\
\geq 1 \text { time a week } \\
\text { Everyday }\end{array}$ \\
\hline
\end{tabular}

CBT, cognitive behavioural therapy.

follow-up). The drop out rates at each visit will be at least below $25 \%$.

- Response rates: the percentage of responses for questions with regards to outcomes at each time.

\section{Procedures}

Figure 1 shows the trial flow chart. Participants will be assessed at baseline (0 week), at weeks 1, 2, 3, 4, 8, 12, 16,20 , and followed up at week 26 after the quit date. The schedule of enrolment and follow-up assessments are summarised in table 5 . The demographic characteristics of gender, age, height and weight (for calculating BMI), educational level (primary, middle school, high school, university and above) will be included. Smoking characteristics of cigarettes per day, quit history, motivation and confidence to quit, quitting smoking gradually or abruptly, nicotine dependence by FTCD and smoking craving by VAS will be included.

Standard abstinence questions will be asked: 'Have you ever smoked during the past 7 days?' and 'Have you ever 


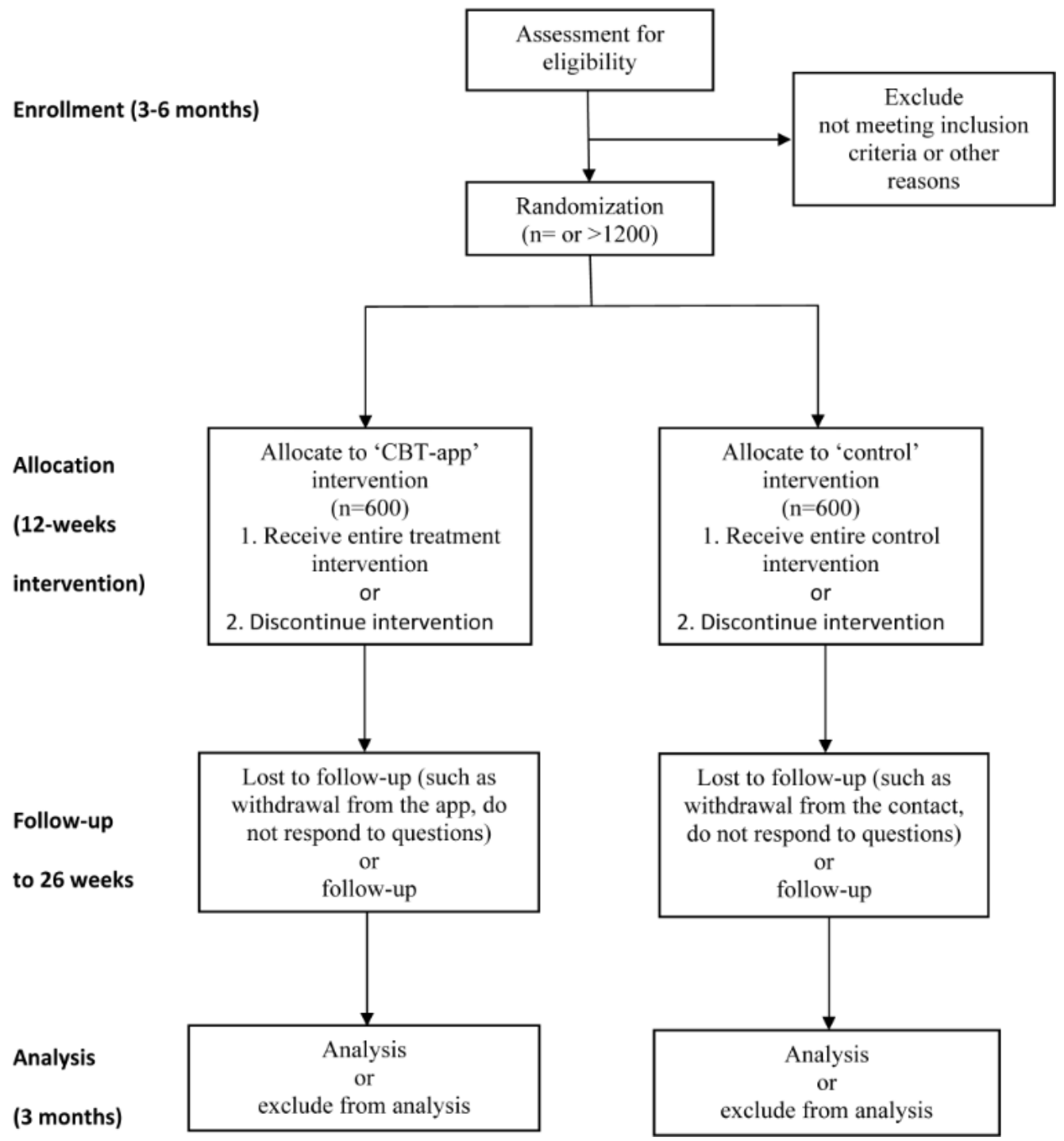

Figure 1 Study flow diagram. CBT,cognitive behavioural therapy.

smoked since (the date of start of abstinence period, that is, from week 2)' A: No, not a puff; B: 1-5 cigarettes; C: More than five cigarettes?' at weeks 1, 2, 3, 4, 8, 12, 16, 20, and at follow-up week 26 after the quit date. If a participant's response is A or B at all points, then a biochemical test will be conducted to confirm the individual as an abstinent. If the response is $\mathrm{C}$, questions regarding cigarette consumption will be asked. Questions about weight gain will be asked monthly until the end of the follow-up week 26 after the quit date. Weight change will be recorded monthly until 6 months postquit. At the end of the trial, participants will be asked, 'Have you used any other smoking cessation methods: such as visit smoking cessation clinics, consulting, smoking cessation medications like nicotine replacement therapy, bupropion, varenicline or any other smoking cessation services or medications'.

\section{Withdrawal from the programme}

Participants will be informed to choose to stay in the app or not without giving any reason. Also, an individual who initially agrees to participate in this study will have the right to withdraw from the study at any point of time and have the right to refuse to answer any particular question(s) or participate in a particular set of procedures in this trial. All participants who still stay in the app will be contacted and not required to provide withdrawal consent, and will be followed up irrespective of their smoking status. Based on the intention-to-treat (ITT) principle, ${ }^{31}$ participants who fail to respond to the follow-up end-point will be retained in the analyses and classified as smokers. However, if participants are unwilling to participate in the study, then they will be excluded from the analyses and the reason(s) for their withdrawal will be recorded if provided.

\section{Data collection}

Questionnaire data will be automatically collected by a data acquisition system into the ePRO Software (http:// www.c-path.org/programs/pro). A user-specified file will be downloaded from the database, and the data will be subjected to basic processing and recoding. There will be only one interim analysis with the 200 participants initially 
Table 5 Schedule of enrolment and follow-up assessments

\begin{tabular}{|c|c|c|}
\hline & Baseline & $\begin{array}{l}\text { Follow-up } \\
\text { weeks }\end{array}$ \\
\hline Initial screen & $\sqrt{ }$ & \\
\hline Informed consent & $\sqrt{ }$ & \\
\hline Eligibility screen & $\sqrt{ }$ & \\
\hline $\begin{array}{l}\text { Demographic and smoking } \\
\text { characteristics }\end{array}$ & $\sqrt{ }$ & \\
\hline $\begin{array}{l}\text { Motivation and confidence in } \\
\text { quitting }\end{array}$ & $\sqrt{ }$ & \\
\hline $\begin{array}{l}\text { Assessment of Nicotine } \\
\text { dependence by FTCD }\end{array}$ & $\sqrt{ }$ & \\
\hline $\begin{array}{l}\text { Randomisation, intervention/ } \\
\text { control initiation }\end{array}$ & $\sqrt{ }$ & \\
\hline Instruction for using app & $\sqrt{ }$ & \\
\hline Cigarette consumption & $\sqrt{ }$ & \\
\hline Weight/weight gain & $\sqrt{ }$ & $\sqrt{ }$ \\
\hline Abstinence & & $\sqrt{ }$ \\
\hline
\end{tabular}

Window period for each data collection date is $-1 \sim+4$ days. FTCD, fagerström test of cigarette dependence.

for assessing the programme acceptability. Only investigators can access data. All data will be monitored by the data monitoring committee of the hospital. Personal data will be deidentified.

\section{Statistical analysis}

On completion of data collection, the data will be cleaned up and prepared for data analysis. The primary and secondary outcome variables and mediating mechanisms will be checked for distribution, outliers and missing patterns and appropriate steps will be taken as and when necessary. All analyses will be conducted based on an ITT. In order to address the non-responses at follow-up, the missing data from lost to follow-up will be expected to be a smoker.

Demographic and smoking-related characteristics will be compared between the groups at baseline using independent t-test for continuous variables or $\chi^{2}$ test for binary variables as appropriate. Logistic regression will be used for between-group comparisons of the primary outcome variable-biologically verified continuous smoking abstinence at week 26 and the secondary outcomes 7-day point prevalence of smoking abstinence (at weeks 1, 2, 3, 4, 8, 12, 16, 20 and 26) and self-reported continuous smoking abstinence (at weeks 4, 8, 12, 16, 20 and 26). For comparisons of secondary continuous outcomes that do not meet the normal distribution assumptions, a non-parametric Mann-Whitney test will be conducted. To account for the non-responses at follow-up, four multiple imputation models will be constructed for the non-responses during the follow-up at 7 days, week 4, week 12 and week 26 of continuous abstinence. The covariates included in the models are age, gender, education level, nicotine dependence and intervention group. Ten imputed data sets will be generated based on Rubin's formula for relative efficiency to produce about $99 \%$ efficiency. ${ }^{32}$ In addition, a complete case analysis will be performed, in which any participant with missing information on any outcome will be excluded. Finally, a subgroup analysis will be conducted for key demographics, smoking and cessation characteristics, social support and use of intervention variables to assess homogeneity of the treatment effects using logistic regression. The effect measures include relative risk and $95 \%$ CIs. Further, a two-sided $p$ value of $<0.05$ will be considered as statistically significant difference. The completed data will be analysed and blinded to the intervention assignment by a trial statistician using the $\mathrm{R}$ software (R Foundation for Statistical Computing, Vienna, Austria. https://www.r-project.org/) and the IBM SPSS Software (IBM, IBM SPSS Statistics for Windows, https:// www.ibm.com/analytics/spss-statistics-software). Also, a sensitivity analysis will be conducted with the assumption that all participants with missing outcome data had not changed the smoking status since the previous follow-up (ie, ITT).

\section{Safety}

Throughout the 'CBT-based smoking cessation app' programme, the participants from both groups will be encouraged to communicate with us (Dr Liao, Dr Tang or research assistants) if they experience any smoking cessation relative health issues. The contact information (available at any time) will be provided to each participant at baseline. Most of the nicotine withdrawal symptoms are only temporary and subside within several weeks. If participants experience severe physical or mental withdrawal symptoms, then they can contact a healthcare provider. These serious symptoms will be recorded in the EDC system.

\section{Ethics and dissemination}

Ethical approval was obtained from the ethics committee of Sir Run Run Shaw Hospital, an affiliate of Zhejiang University, Medical College (number: 20200129-33). The trial will be performed in accordance with the Declaration of Helsinki. Informed consent will be obtained from all the participants. Each participant will be explained with regards to the purpose, procedures and measurements, potential risks and benefits of the trial before recruitment. All the obtained data will be deidentified and cannot be linked back to the participants. The Unique Identifiers (UIDs) will be automatically deleted from the users' devices at the end of the trial. The study data collection will start from January 2021. Only the principal investigator and researcher assistants have access to the study data. The primary and secondary analyses will be started immediately after completion of the trial. The results of the trial will be published in the conference proceedings and peerreviewed journals according to the Consolidated Standards of Reporting Trials statement. 


\section{DISCUSSION}

This trial will investigate the effectiveness of smartphone apps developed for smoking cessation in China, with the primary outcome of biologically verified continuous smoking abstinence at 6 months, as recommended by the SRNT ${ }^{26}$ and the 'Russell Standard'. ${ }^{27}$ To our knowledge, this will be the first RCT to evaluate the efficacy of smoking cessation intervention by using an app based on the cognitive behavioural principles and empirical evidence in Mainland China.

The strength of this study is that it is the first theoretical frameworks guided (CBT), and evidence-based (on the basis of clinical experience, scientific or empirical evidence of smoking cessation) RCT that adheres to the guideline recommendations to evaluate the smoking cessation app in Mainland China. In an era where the prevalence of smartphones and their associated apps have been shown, the trend of exploring and applying these apps in promoting health behaviours such as smoking cessation has become important and inevitable. Smartphone-based smoking cessation apps were easily accessible, highly tailored, intensive but flexible and inexpensive or free of charge. If this app programme is proved to be effective (the funder will further provide financial support to run the app), it opens up a huge potential use in China, with a country of more than 300 million smokers, at the population level, and the impact of public health could be enormous.

The weaknesses of this study include abstinence, which will be mainly measured via a self-report through the app questionnaires, and this is less rigorous than a biochemically verified abstinence. Thus, biological verification for those who have self-reported continuous smoking abstinence at 6 months should be provided and chose this outcome as the primary outcome in this study. In terms of biochemically verified abstinence, participants will receive urine cotinine test by mail (the test results will be confirmed by video call). In order to assess the efficacy of only CBT-based smoking cessation interventions by app, another limitation will be that no first-line medications will be provided to the participants. Furthermore, the participants in this study might be more likely motivated than other smokers, and they might search for other smoking cessation apps or any other available smoking cessation services during the trial. However, the resources that are designed to help people quit smoking and remain smoke-free are extremely limited in China. ${ }^{6}$ Finally, participants may be more likely to drop out from the trial, as this smoking cessation app programme will only provide online services. Thus, participants should be encouraged to contact us in case of any question. Also, an ITT approach will be applied to increase the precision and reduce the bias by missing data from loss to follow-up participants.

Text messaging on mobile phones has already been efficacious in helping people to quit smoking in China. ${ }^{7}$ Smartphones offer the possibility of supplementing text message-based interventions with computer programmes that can weave together with expert systems, multimedia (eg, music, audio or video), and the internet (eg, WeChat, Weibo or other social networking sites). However, research on content analysis revealed that higher star ratings showed association with better adherence to the 5 As. But most of the currently available smartphone apps for smoking cessation are not particularly 'smart', as they have low levels of adherence to the guidelines. ${ }^{25} 33$ This new app has been designed and will be revised according to the evidence on smoking cessation principles. This trial assists in building our understanding of how this CBTbased smoking cessation app can be effective in helping people to quit smoking in China.

In summary, this is the first RCT to evaluate the efficacy of CBT-based cigarette smoking cessation smartphone app in China. If this is approved to be effective, then its use will help bridge the huge gap between the unmet needs and the demands for smoking cessation in China at population-based level.

Acknowledgements Dr Liao would like to thank Ge Zhang, Huimin Wang, Roland Perfekt, Tobias Danielsson, Tom Wu, Jennifer Yang from Johnson \& Johnson pharmaceutical company for providing critical comments and suggestions for the study design, thank Johnson \& Johnson pharmaceutical company for developing the app.

Contributors $\mathrm{YL}$ and JT developed and designed the study. YL took the lead in drafting the manuscript protocol with contributions by JT advised the study design, and coordinated study approval. JT read and proposed critical comments, as well as approved the manuscript for publication.

Funding The research is supported by Johnson \& Johnson pharmaceutical company (K-20201478). The funder will collaboratively involve in the study design, development of data collection tool, App software management, but company will have no role in involving in enrolment, providing informed consent form, contacting with participants, addressing safety issues, monitoring and collecting data, writing the manuscript and submitting the paper for publication.

Competing interests Dr Liao received funding from Johnson \& Johnson pharmaceutical company for the study. Dr Tang has no potential conflicts of interest to declare.

Patient consent for publication Not required.

Provenance and peer review Not commissioned; externally peer reviewed.

Supplemental material This content has been supplied by the author(s). It has not been vetted by BMJ Publishing Group Limited (BMJ) and may not have been peer-reviewed. Any opinions or recommendations discussed are solely those of the author(s) and are not endorsed by BMJ. BMJ disclaims all liability and responsibility arising from any reliance placed on the content. Where the content includes any translated material, BMJ does not warrant the accuracy and reliability of the translations (including but not limited to local regulations, clinical guidelines, terminology, drug names and drug dosages), and is not responsible for any error and/or omissions arising from translation and adaptation or otherwise.

Open access This is an open access article distributed in accordance with the Creative Commons Attribution Non Commercial (CC BY-NC 4.0) license, which permits others to distribute, remix, adapt, build upon this work non-commercially, and license their derivative works on different terms, provided the original work is properly cited, appropriate credit is given, any changes made indicated, and the use is non-commercial. See: http://creativecommons.org/licenses/by-nc/4.0/.

\section{ORCID iD}

Yanhui Liao http://orcid.org/0000-0003-4735-3252

\section{REFERENCES}

1 Liu S, Zhang M, Yang L, et al. Prevalence and patterns of tobacco smoking among Chinese adult men and women: findings of the 
2010 national smoking survey. J Epidemiol Community Health 2017;71:154-61.

2 Li Q, Hsia J, Yang G. Prevalence of smoking in China in 2010. N Engl J Med Overseas Ed 2011;364:2469-70.

3 Li S, Meng L, Chiolero A, et al. Trends in smoking prevalence and attributable mortality in China, 1991-2011. Prev Med 2016;93:82-7.

4 Tan X, Liu X, Shao H, et al. A vision for health care. Value in health regional issues 2030;2017:112-4.

5 GATS. Global youth tobacco survey, 2018. Available: https://www. tobaccofreekids.org/assets/global/pdfs/en/GATS_China_2018_ FactSheet.pdf

6 Lin H, Xiao D, Liu Z, et al. National survey of smoking cessation provision in China. Tob Induc Dis 2019;17:25.

7 Liao Y, Wu Q, Kelly BC, et al. Effectiveness of a text-messagingbased smoking cessation intervention ("Happy Quit") for smoking cessation in China: A randomized controlled trial. PLoS Med 2018;15:e1002713.

8 Abroms LC, Lee Westmaas J, Bontemps-Jones J, et al. A content analysis of popular smartphone apps for smoking cessation. Am J Prev Med 2013;45:732-6.

9 Vilardaga R, Casellas-Pujol E, McClernon JF, et al. Mobile applications for the treatment of tobacco use and dependence. Curr Addict Rep 2019;6:86-97.

10 Whittaker R, McRobbie H, Bullen C, et al. Mobile phone text messaging and app-based interventions for smoking cessation. Cochrane Database Syst Rev 2019;17.

11 Clinical Practice Guideline Treating Tobacco Use and Dependence 2008 Update Panel, Liaisons, and Staff. A clinical practice guideline for treating tobacco use and dependence: 2008 update. A U.S. public health service report. Am J Prev Med 2008;35:158-76.

12 Cheng F, Xu J, Su C, et al. Content analysis of smartphone apps for smoking cessation in China: empirical study. JMIR Mhealth Uhealth 2017;5:e93.

13 Patel R, Sulzberger L, Li G, et al. Smartphone apps for weight loss and smoking cessation: quality ranking of 120 apps. N Z Med J 2015;128:73.

14 Jacobs MA, Cobb CO, Abroms L, et al. Facebook apps for smoking cessation: a review of content and adherence to evidence-based guidelines. J Med Internet Res 2014;16:e205.

15 Bricker JB, Mull KE, Kientz JA, et al. Randomized, controlled pilot trial of a smartphone APP for smoking cessation using acceptance and commitment therapy. Drug Alcohol Depend 2014;143:87-94.

16 Villanti AC, West JC, Klemperer EM, et al. Smoking-Cessation interventions for U.S. young adults: updated systematic review. Am J Prev Med 2020;59:123-36.

$17 \mathrm{Xu}$ J, Bricker J, Fu X, et al. Design and development of smoking cessation Apps based on smokers' and providers' perspectives in China: survey study. JMIR Mhealth Uhealth 2019;7:e12200.
18 Heishman SJ. Behavioral and cognitive effects of smoking: relationship to nicotine addiction. Nicotine Tob Res 1999;1 Suppl 2:S143-7.

19 Beck AT. Cognitive therapy. A 30-year retrospective. Am Psychol 1991;46:368-75.

20 Hofmann SG, Asnaani A, Vonk IJJ, et al. The efficacy of cognitive behavioral therapy: a review of meta-analyses. Cognit Ther Res 2012;36:427-40.

21 Stead LF, Carroll AJ, Lancaster T. Group behaviour therapy programmes for smoking cessation. Cochrane Database Syst Rev 2017;3:CD001007.

22 Webb MS, de Ybarra DR, Baker EA, et al. Cognitive-behavioral therapy to promote smoking cessation among African American smokers: a randomized clinical trial. J Consult Clin Psychol 2010;78:24-33.

23 Perkins KA, Marcus MD, Levine MD, et al. Cognitive-behavioral therapy to reduce weight concerns improves smoking cessation outcome in weight-concerned women. J Consult Clin Psychol 2001;69:604-13.

24 Brown RA, Kahler CW, Niaura R, et al. Cognitive-behavioral treatment for depression in smoking cessation. J Consult Clin Psychol 2001;69:471-80.

25 Hoeppner BB, Hoeppner SS, Seaboyer L, et al. How smart are smartphone apps for smoking cessation? A content analysis. Nicotine Tob Res 2016;18:1025-31.

26 Hughes JR, Keely JP, Niaura RS, et al. Measures of abstinence in clinical trials: issues and recommendations. Nicotine Tob Res 2003;5:13-26.

27 West R, Hajek P, Stead L, et al. Outcome criteria in smoking cessation trials: proposal for a common standard. Addiction 2005;100:299-303.

28 Huang $\mathrm{C}-\mathrm{L}$, Lin $\mathrm{H}-\mathrm{H}$, Wang $\mathrm{H}-\mathrm{H}$. The psychometric properties of the Chinese version of the Fagerstrom test for nicotine dependence. Addict Behav 2006;31:2324-7.

29 Heatherton TF, Kozlowski LT, Frecker RC, et al. The Fagerström test for nicotine dependence: a revision of the Fagerström tolerance questionnaire. Br J Addict 1991;86:1119-27.

30 Fagerström K. Determinants of tobacco use and renaming the FTND to the Fagerstrom test for cigarette dependence. Nicotine Tob Res 2012;14:75-8.

31 Montori VM, Guyatt GH. Intention-To-Treat principle. CMAJ 2001;165:1339-41.

32 Rubin DB. Multiple imputation for nonresponse in surveys. Hoboken: John Wiley \& Sons, 2004.

33 Abroms LC, Padmanabhan N, Thaweethai L, et al. iPhone apps for smoking cessation: a content analysis. Am J Prev Med 2011;40:279-85. 